\title{
Fine Structure of the Hydrogen Atom by a Microwave Method***
}

\author{
Willis E. Lamb, JR. and Robert C. Retherford \\ Columbia Radiation Laboratory, Department of Physics, Columbia University, New York, New York
}

(Received June 18, 1947)

$T$ HE spectrum of the simplest atom, hydrogen, has a fine structure ${ }^{1}$ which according to the Dirac wave equation for an electron moving in a Coulomb field is due to the combined effects of relativistic variation of mass with velocity and spin-orbit coupling. It has been considered one of the great triumphs of Dirac's theory that it gave the "right" fine structure of the energy levels. However, the experimental attempts to obtain a really detailed confirmation through a study of the Balmer lines have been frustrated by the large Doppler effect of the lines in comparison to the small splitting of the lower or $n=2$ states. The various spectroscopic workers have alternated between finding confirmation ${ }^{2}$ of the theory and discrepancies ${ }^{3}$ of as much as eight percent. More accurate information would clearly provide a delicate test of the form of the correct relativistic wave equation, as well as information on the possibility of line shifts due to coupling of the atom with the radiation field and clues to the nature of any non-Coulombic interaction between the elementary particles: electron and proton.

The calculated separation between the levels $2^{2} P_{1}$ and $2^{2} P_{3 / 2}$ is $0.365 \mathrm{~cm}^{-1}$ and corresponds to a wave-length of $2.74 \mathrm{~cm}$. The great wartime advances in microwave techniques in the vicinity of three centimeters wave-length make possible the use of new physical tools for a study of the $n=2$ fine structure states of the hydrogen atom. A little consideration shows that it would be exceedingly difficult to detect the direct absorption of radiofrequency radiation by excited $\mathrm{H}$ atoms in a gas discharge because of their small

* Publication assisted by the Ernest Kempton Adams Fund for Physical Research of Columbia University, New York.

** Work supported by the Signal Corps under contract number W 36-039 sc-32003.

${ }^{1}$ For a convenient account, see H. E. White, Introduction to Atomic Spectra (McGraw-Hill Book Company, New York, 1934), Chap. 8.

2 J. W. Drinkwater, O. Richardson, and W. E. Williams, Proc. Roy. Soc. 174, 164 (1940).

${ }^{3}$ W. V. Houston, Phys. Rev. 51, 446 (1937); R. C. Williams, Phys. Rev. 54, 558 (1938); S. Pasternack, Phys.

Rev. 54, 1113 (1938) has analyzed these results in terms of an upward shift of the $S$ level by about $0.03 \mathrm{~cm}^{-1}$. population and the high background absorption due to electrons. Instead, we have found a method depending on a novel property of the $2^{2} S_{3}$ level. According to the Dirac theory, this state exactly coincides in energy with the $2^{2} P_{\frac{1}{3}}$ state which is the lower of the two $P$ states. The $S$ state in the absence of external electric fields is metastable. The radiative transition to the ground state $1^{2} S_{\frac{1}{3}}$ is forbidden by the selection rule $\Delta L= \pm 1$. Calculations of Breit and Teller ${ }^{4}$ have shown that the most probable decay mechanism is double quantum emission with a lifetime of $1 / 7$ second. This is to be contrasted with a lifetime of only $1.6 \times 10^{-9}$ second for the nonmetastable $2^{2} P$ states. The metastability is very much reduced in the presence of external electric fields ${ }^{5}$ owing to Stark effect mixing of the $S$ and $P$ levels with resultant rapid decay of the combined state. If for any reason, the $2^{2} S_{\frac{1}{2}}$ level does not exactly coincide with the $2^{2} P_{\frac{1}{2}}$ level, the vulnerability of the state to external fields will be reduced. Such a removal of the accidental degeneracy may arise from any defect in the theory or may be brought about by the Zeeman splitting. of the levels in an external magnetic field.

In brief, the experimental arrangement used is the following: Molecular hydrogen is thermally dissociated in a tungsten oven, and a jet of atoms emerges from a slit to be cross-bombarded by an electron stream. About one part in a hundred million of the atoms is thereby excited to the metastable $2^{2} S_{\frac{1}{3}}$ state. The metastable atoms (with a small recoil deflection) move on out of the bombardment region and are detected by the process of electron ejection from a metal target. The electron current is measured with an FP-54 electrometer tube and a sensitive galvanometer.

If the beam of metastable atoms is subjected to any perturbing fields which cause a transition to any of the $2^{2} P$ states, the atoms will decay while moving through a very small distance. As a result, the beam current will decrease, since the

${ }^{4} \mathrm{H}$. A. Bethe in Handbuch der Physik, Vol. 24/1, $\$ 43$.

${ }^{5}$ G. Breit and E. Teller, Astrophys. J. 91, 215 (1940). 


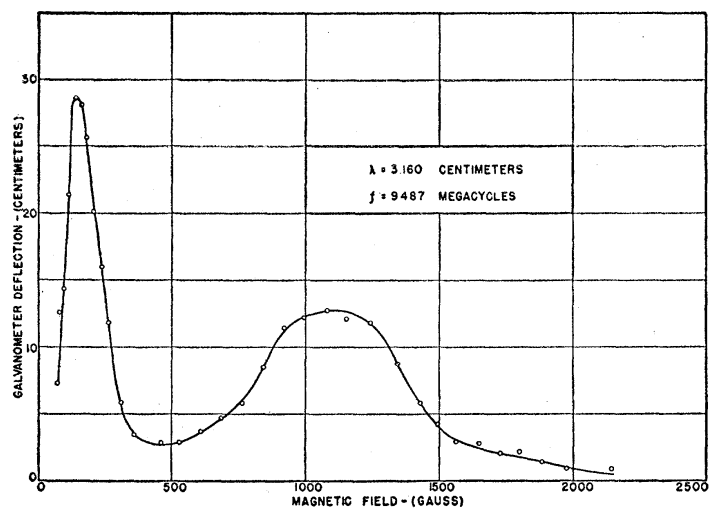

FIG. 1. A typical plot of galvanometer deflection due to interruption of the microwave radiation as a function of magnetic field. The magnetic field was calibrated with a flip coil and may be subject to some error which can be largely eliminated in a more refined apparatus. The width of the curves is probably due to the following causes: (1) the radiative line width of about $100 \mathrm{Mc} / \mathrm{sec}$. of the ${ }^{2} P$ states, (2) hyperfine splitting of the ${ }^{2} S$ state which amounts to about $88 \mathrm{Mc} / \mathrm{sec}$., (3) the use of an excessive intensity of radiation which gives increased absorption in the wings of the lines, and (4) inhomogeneity of the magnetic field. No transitions from the state $2^{2} S_{\frac{1}{3}}\left(m=-\frac{1}{2}\right)$ have been observed, but atoms in this state may be quenched by stray electric fields because of the more nearly exact degeneracy with the Zeeman pattern of the ${ }^{2} P$ states.

detector does not respond to atoms in the ground state. Such a transition may be induced by the application to the beam of a static electric field somewhere between source and detector. Transitions may also be induced by radiofrequency radiation for which $h \nu$ corresponds to the energy difference between one of the Zeeman components of $2^{2} S_{\frac{1}{3}}$ and any component of either $2^{2} P_{\frac{1}{2}}$ or $2^{2} P_{3 / 2}$. Such measurements provide a precise method for the location of the $2^{2} S_{1}$ state relative to the $P$ states, as well as the distance between the latter states.

We have observed an electrometer current of the order of $10^{-14}$ ampere which must be ascribed to metastable hydrogen atoms. The strong quenching effect of static electric fields has been observed, and the voltage gradient necessary for this has a reasonable dependence on magnetic field strength.

We have also observed the decrease in the beam of metastable atoms caused by microwaves in the wave-length range 2.4 to $18.5 \mathrm{~cm}$ in various magnetic fields. In the measurements, the frequency of the $r-f$ is fixed, and the change in the galvanometer current due to interruption of the $\mathrm{r}-\mathrm{f}$ is determined as a function of magnetic field

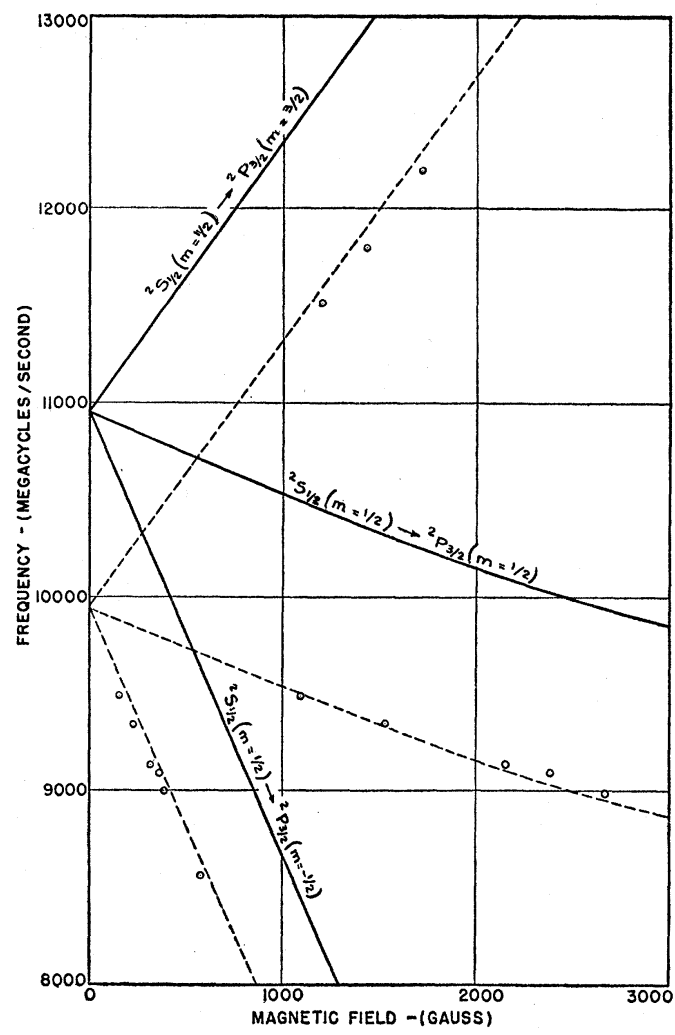

FIG. 2. Experimental values for resonance magnetic fields for various frequencies are shown by circles. The solid curves show three of the theoretically expected variations, and the broken curves are obtained by shifting these down by $1000 \mathrm{Mc} / \mathrm{sec}$. This is done merely for the sake of comparison, and it is not implied that this would represent a "best fit." The plot covers only a small range of the frequency and magnetic field scale covered by our data, but a complete plot would not show up clearly on a small scale, and the shift indicated by the remainder of the data is quite compatible with a shift of $1000 \mathrm{Mc}$.

strength. A typical curve of quenching versus magnetic field is shown in Fig. 1. We have plotted in Fig. 2 the resonance magnetic fields for various frequencies in the vicinity of 10,000 $\mathrm{Mc} / \mathrm{sec}$. The theoretically calculated curves for the Zeeman effect are drawn as solid curves, while for comparison with the observed points, the calculated curves have been shifted downward by $1000 \mathrm{Mc} / \mathrm{sec}$. (broken curves). The results indicate clearly that, contrary to theory but in essential agreement with Pasternack's hypothesis, ${ }^{3}$ the $2^{2} S_{1}$ state is higher than the $2^{2} P_{\frac{1}{3}}$ by about $1000 \mathrm{Mc} / \mathrm{sec}$. $\left(0.033 \mathrm{~cm}^{-1}\right.$ or about 9 percent of the spin relativity doublet separation. The lower frequency transitions ${ }^{2} S_{\mathfrak{\xi}}\left(m=\frac{1}{2}\right) \rightarrow$ ${ }^{2} P_{1}\left(m= \pm \frac{1}{2}\right)$ have also been observed and agree 
well with such a shift of the ${ }^{2} S_{1}$ level. With the present precision, we have not yet detected any discrepancy between the Dirac theory and the doublet separation of the $P$ levels. (According to most of the imaginable theoretical explanations of the shift, the doublet separation would not be affected as much as the relative location of the $S$ and $P$ states.) With proposed refinements in sensitivity, magnetic field homogeneity, and calibration, it is hoped to locate the $S$ level with respect to each $P$ level to an accuracy of at least ten $\mathrm{Mc} / \mathrm{sec}$. By addition of these frequencies and assumption of the theoretical formula $\Delta \nu=\frac{1}{16} \alpha^{2} R$ for the doublet separation, it should be possible to measure the square of the fine structure constant times the Rydberg frequency to an accuracy of 0.1 percent.

By a slight extension of the method, it is hoped to determine the hyperfine structure of the $2^{2} S_{\mathbf{i}}$ state. All of these measurements will be repeated for deuterium and other hydrogen-like atoms.

A paper giving a fuller account of the experimental and theoretical details of the method is being prepared, and this will contain later and more accurate data.

The experiments described here were discussed at the Conference on the Foundations of Quantum Mechanics held at Shelter Island on June 1-3, 1947 which was sponsored by the National Academy of Sciences.

\title{
On Self-Quenching Halogen Counters
}

\author{
R. D. Present* \\ University of Tennessee, Knoxville, Tennessee
}

(Received May 23, 1947)

$S^{o}$ OME considerations relating the spectroscopic and photochemical properties of polyatomic gases with their use in Geiger-Mueller counters have been developed by the writer. ${ }^{1}$ The selfquenching counter is able to operate without the usual large resistance because of the absence of secondary emission of electrons when the positive ion sheath reaches the cathode and is neutralized. The neutralization of the ions (at about $10^{-7} \mathrm{~cm}$ from the wall) results in neutral atoms or molecules having an excitation energy of $(I-\varphi)$ approximately, where $I$ is the ionization energy and $\varphi$ the photoelectric work function of the surface. In the case of a monatomic or diatomic (non-quenching) gas this excitation energy is transferred to the metal in about $10^{-12}$ sec. and secondary electrons are liberated if $I>2 \varphi$. In the case of most polyatomic gases (with four or more atoms), the neutralized molecules will predis-

* Also consultant at Clinton Laboratories, Oak Ridge, Tennessee.

1S. A. Korff and R. D. Present, Phys. Rev. 65, 274 (1944). The theoretical considerations in this paper were mainly developed by R. D. Present; the experimental work and its interpretation were done mainly by S. A. Korff. sociate (in about $10^{-13} \mathrm{sec}$.) before they can make an inelastic collision with the wall, and the emission of secondary electrons will be negligible. In order to determine whether a given gas will show self-quenching action, it is sufficient to examine its absorption spectrum at wave-lengths corresponding to an excitation energy of $(I-\varphi)$. Continuous absorption, or a line broadening corresponding to a predissociation lifetime of $10^{-13}$ sec., indicate that the molecule undergoes primary photo-decomposition. Such a gas will make a self-quenching counter. If a mixture of quenching and non-quenching gases is used, the counter will show self-quenching action only if the ionization potential of the non-quenching gas exceeds that of the quenching gas. Under these circumstances electron transfer changes the composition of the positive ion sheath so that, when it reaches the cathode, there is a negligible number of non-quenching ions. A more detailed discussion will be found in reference 1 .

We should like to point out here that the mechanism of secondary-emission quenching is compatible, under special circumstances, with the 